\title{
Anatomically Guided Registration of Whole Body Mouse MR Images
}

\author{
N. Kovacevic ${ }^{1}$, G. Hamarneh ${ }^{1}$, and M. Henkelman ${ }^{1,2}$ \\ ${ }^{1}$ Mouse Imaging Centre, Hospital For Sick Children \\ 555 University Ave., Toronto ON M5G 1X8, Canada \\ ${ }^{2}$ University Of Toronto, Canada
}

\begin{abstract}
A new methodology for three-dimensional (3D) non-linear registration of articulate objects is presented. This approach is described in the context of a program to screen for mutant mouse phenotypes based on high resolution MR images of whole animals. The screening process requires statistical definition of the normal (non-mutant) mouse anatomy and its variation. The registration algorithm is designed to remove postural differences between mice and thus enable meaningful comparisons between images. The main focus of our approach is anatomical content and its preservation under deformations. Prior knowledge guides the registration through an evolutionary process based on a hierarchical tree of mouse anatomy.
\end{abstract}

\section{Introduction}

The comprehensive study of the biological functions of genes has lead a number of centers around the world to start large scale mouse mutagenesis projects (Jackson Laboratory, Mouse Genome Centre at Harwell, RIKEN Genomics Sciences Center, to name the few). At CMHD, The Centre For Modeling Human Disease at Mount Sinai Hospital in Toronto, a genome-wide random mutagenesis is used for discovering new genes and creating new mouse models of human diseases. A set of primary physiological and behavioral screens is designed to parse large numbers of mice and identify those with unusual phenotypes. Most of the primary tests are designed to screen for human-like diseases and are tissue or organ specific (for example, heart rate, blood chemistry, glucose tolerance test, vision and hearing). High resolution, whole body MR imaging presents a powerful tool that can help in the assessment of gross morphology (e.g., sizes, shapes and textures of organs and tissues). It has opened a new area of research into MRI based mouse phenotyping. In a high-throughput setting, however, the radiological evaluation of large MR images (over $0.5 \mathrm{~GB}$ per mouse) is a difficult task. In the presence of postural differences, for example, comparing organ anatomy between animals can be both time consuming and unreliable. It is essential to develop algorithms for computer aided morphological phenotyping.

Inter subject comparisons require 3D registration as a way of establishing a correspondence between the same anatomical structures. In the most general setting, fully automatic registration could be an intractable problem. We work with a strictly controlled experiment, however: all mice are taken from the same inbred strain, same sex and same age. Uniform genetic background guarantees small phenotypical variations as 
measured by the standard screens (for example, see The Jackson Laboratory Genome database). Even though there are no screens for the systematic anatomical evaluation, the available data indicates that the intra-strain gross moprhology should be uniform as well. Therefore, the main difficulty for 3D registration lies in postural differences. Image registration, if successful, will in turn enable a formalized, statistical evaluation of the gross, strain and sex specific, morphological variability.

The underlying physical deformation for the whole mouse body is remarkably complex and cannot be modeled in a uniform way. For example, the variation in head position is well modeled by affine transformations, while inner, somewhat amorphous organs like the liver require elastic or free-form deformations. Using elastic or spline based matching algorithms on the entire body would lead to physically implausible results. An additional complication comes from intestines with their complicated, highly variable geometry, even across a single strain. They are also susceptible to many extrinsic factors due to digestion and metabolism. Registering intestines themselves is not only impractical, but their richness with features can be misleading to any registration algorithm.

Numerous automatic and semi-automatic methods for non-rigid registration have been developed and successfully applied to medical images. A large collection of medical image registration techniques is given in [4]. Human brain warping algorithms are reviewed in [1]. Existing methods have been developed for registering specific human organs or tissues and are not directly applicable to whole body MRI's. They typically involve a considerable number of image specific preprocessing steps. For example, intensity-based algorithms require precise masks outlining structures of interest, while model-based algorithms require feature extraction (e.g., curves or polygons are used for modeling of anatomical structures). Manual or semi-automatic procedures are often used, which is unacceptable in our high-throughput framework.

In view of the above considerations, we propose an organ or a region of interest (ROI) based approach to mouse anatomy. We consider the mouse body as a collection of ROI's, and we model its posture using piecewise affine transformations. Our registration method is fully automatic, hierarchical and anatomically guided. Prior anatomical knowledge is encoded in a single image as a reference resource. Deformation fields produced by the algorithm enable transfer of the anatomical knowledge to all other images. This type of registration has many possible applications. Registration based segmentation, though somewhat inaccurate due to the limitations of locally affine transformations, can provide a starting point for more sophisticated organ segmentations. Another application will be in enabling quick ROI browsing of images. For example, if a researcher is interested in the mouse heart, then its approximate location and segmentation will be available from the database, which records images together with their corresponding deformation fields. All mouse hearts form the database could then be resampled into the same coordinate space for easy simultaneous viewing and comparison.

\section{Registration Method}

\subsection{Standard Coordinate Space and Labeling}

We select an image of a representative member of the population, and we refer to it as the reference image. The reference image is considered as the target for all registrations. 
Given an arbitrary image from the mouse population, which we refer to as a sample image, we must describe a transformation which brings it into alignment with the reference image. The alignment must ensure correspondence between anatomical structures, e.g., the 5-th vertebra of the sample mouse must be aligned with the 5-th vertebra of the reference mouse. This is an important point: simple evaluation of some similarity function based on image intensities and/or features without awareness of the underlying anatomy is not sufficient. For this reason prior anatomical knowledge about the reference image is obtained first and subsequently used for guiding the registration process. Anatomical knowledge about the sample image gradually emerges and improves as the registration process evolves.

Prior anatomical knowledge is encoded via manual or semi-automatic labeling of the entire reference image. More precisely, each voxel in the reference image is assigned to one of a finite number of anatomical labels or to the background. The labels represent distinct structures of similar volume (e.g, one label for each vertebra). Particular care is taken in the delineation along the boundaries between structures that can assume independent positions (e.g., femur and pelvis). As mentioned earlier, intestines are best avoided in the registration, so all voxels in this region are assigned to the background. The parcelation of the reference mouse thus obtained is considered to be on the finest scale, with the largest number of labels. The next subsection explains how other, coarser parcelations are derived in a hierarchical manner.

\subsection{Hierarchical Anatomical Tree}

Starting from the finest scale labeling we build a hierarchical tree based on a "partof" concept. The tree consists of hierarchically organized labelings $L_{0}, L_{1}, \ldots, L_{N}$. The finest scale labeling, $L_{N}$, is the manual one described above. Its parent labeling, $L_{N-1}$, is obtained by merging together labels of $L_{N}$. For example, if $L_{N}$ has separate labels for each of the heart chambers (total of 4 labels), then $L_{N-1}$ may have only two labels: the two ventricles are merged into one label and the two atria are merged into another. As a result, $L_{N-1}$ is a coarser labeling i.e., it has fewer labels but they encompass larger volumes. The next coarser labeling $L_{N-2}$ is obtained by merging together labels of $L_{N-1}$ and so on. The coarsest labeling $L_{0}$ contains a single label encompassing the whole reference mouse body (Fig. 1 .

\subsection{Hierarchical Registration}

The registration is designed as an evolutionary process which follows the tree hierarchy. It starts with the alignment of the single label of $L_{0}$ and ends with the piecewise alignment of $L_{N}$. The transformations produced in the process are piecewise affine, where the pieces correspond to labels, and are expressed as vector fields.

We begin with the affine alignment of the single label of $L_{0}$ with the sample image. This step accounts for global rotations, translations, shears and scalings. Next, we descend to the $L_{1}$ level. The labels of $L_{1}$ are independently aligned with the sample image, again using an affine transformation model. The transformation of the single parent label of $L_{0}$ obtained in the previous step is used to initialize all $L_{1}$ label alignments. The descent to $L_{2}$ proceeds in a similar fashion: the alignments of $L_{2}$ labels are initialized with 

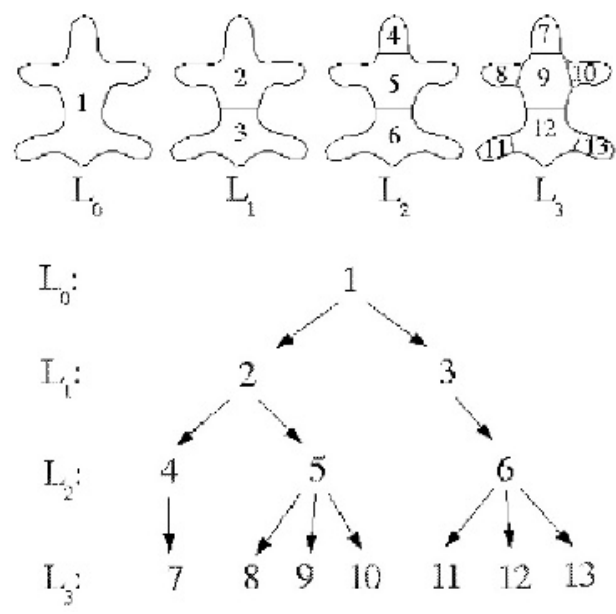

Fig. 1. Hierarchy of labelings $L_{0}, L_{1}, \ldots, L_{N}$. Arrows represent parent $\rightarrow$ child relationship based on a "part-of" concept

the transformations of their $L_{1}$ parents. The process continues until the finest labeling $L_{N}$ is reached.

Label specific transformations of $L_{N}$ are gathered to form a vector field, $V_{N}$ : for each voxel in the reference image, we apply the transformation of its $L_{N}$ label to calculate the corresponding point in the sample image. The difference between the two spatial coordinates is encoded as a 3D displacement vector (Fig. (2).

Having in mind that our main goal is a general anatomical correspondence for ROI based comparisons, we measure the success of the registration accordingly: for each label of $L_{N}$, we compare the corresponding ROI's in the reference image and the $V_{N^{-}}$ transformed sample image respectively; if visual inspection confirms that the two ROI's encompass the same anatomical structure, then the registration is considered a success.

\section{Implementation}

We have implemented the registration algorithm in view of its future applications to phenotypical screening. The implementation assumes that: (i) the same MR sequence is used on all mice (ii) the morphological variation between subjects is small and (iii) the postural differences are moderate (a general body posture is controlled by positioning mice on specially designed platforms). These assumptions impact on the registration schedule because they imply a high level of confidence in passing transformations from parent labels to their children.

We encode the registration schedule as a list of level-specific protocol files which control single label alignments. Suppose $C$ is a label at level $L_{i}$ and $P$ is its parent at level $L_{i-1}$. Assume that $P$ has been aligned previously, resulting in an affine transformation $T_{P}$. In order to align $C$, we use $T_{P}$ in two ways: (i) as an initial transformation and (ii) to back-transform $C$ label to the sample image. This puts us 


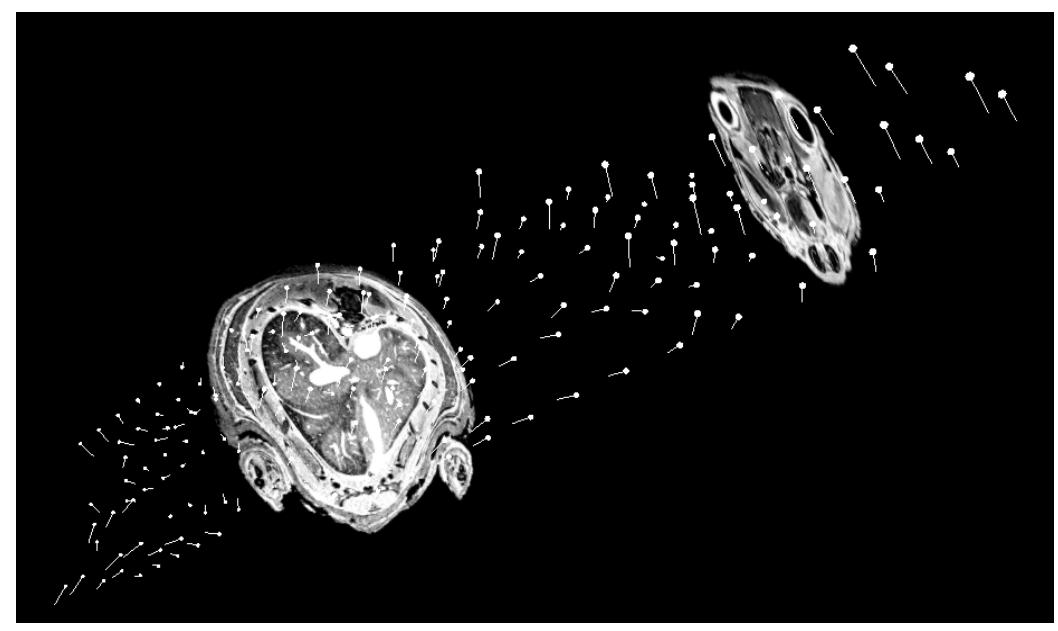

Fig. 2. Final displacement field $V_{N}$ depicted with two cross-sections of the reference mouse overlaid to provide spatial context. Shown is a sparse, regular grid of points in the reference space (white dots) with displacement vectors (white lines) pointing toward their corresponding points in the sample space

in a standard registration framework: target image and target mask (defined by $C$ ) vs. source image and source mask (defined by the transformed $C$ label). The source mask is only an approximation and may not include all of the required anatomy. Therefore, we dilate the source mask by a level-specific amount, encoded in the protocol file of $L_{i}$ (Fig. 3). The dilation amounts decrease from one level to the next, reflecting an increasing confidence in registration accuracy. Protocol files also encode parameters for multi-resolution and multi-scale alignment schedules. Similarly to [3], the schedules prescribe successive use of downsampled, smoothed and/or edge detected images, together with pyramidal sampling parameters and the number of iterations for each succession. Appropriate values for the parameters are determined experimentally (an example is given in the Results section). Note that the alignments of labels on the same hierarchical level are mutually independent, i.e., they only depend on their parent transformations. The algorithm takes advantage of this fact via parallelization.

We have chosen the affine alignment of AIR5.2.2 ([6]) as our core alignment algorithm for its computational efficiency and robustness. In accordance to the same imaging modality assumption, we have used the scaled least square difference as the similarity function with the Levenberg-Marquardt-like optimization ([6]).

\section{Results}

We have evaluated the performance of the algorithm in a synthetic experiment. As a reference image we selected a full mouse body MR image (2.0T GE Medical Systems console, perfused with 20:1 magnevist to $10 \%$ buffered formalin, 3D spin-echo TR/TE = 

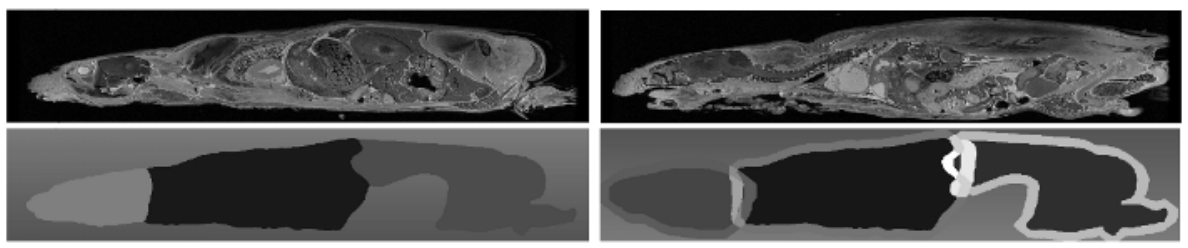

Fig. 3. Example: labeling $L_{1}$ on the reference image (top-left) consists of 3 labels (bottom-left). Corresponding approximate labels (bottom-right) in the sample image (top-right) are dilated by a lavel-specific amount to ensure inclusion of the appropriate anatomy

$100 \mathrm{~ms} / 6.552 \mathrm{~ms}, 90 \mathrm{flip}$, averages=16, 1024x256x256 acquisition matrix, $118 \times 29 \times 29$ $\mathrm{mm}$ fov, for more details see [7]). From this image we created a second, synthetic image to be used as the sample image. We picked 14 landmarks in the reference image and displaced them arbitrarily in 3D space. The average magnitude of the landmark displacement vectors was $1.8 \mathrm{~mm}$ (approx. 16 voxels). Based on the landmark displacements, we next transformed the entire reference image using thin-plate splines ([2]). To create a more realistic scenario, we added gaussian noise (standard deviation of noise $=9 \%$ of mean signal intensity) to the deformed image and the result was used as a sample image. We manualy created 66 labels on the reference image as the finest scale labeling, and from it a hierarchical tree composed of five levels, with five corresponding registration protocols.

Table 1. Details of $L_{1}$ registration protocol. Sampling schedule in the second column refers to subsampling of images using a regular grid with nodal distance given in $\mathrm{mm}$ 's. Two sampling distances indicate a two-step registration, going from coarser to finer image subsampling. Third column specifies dilation amounts applied to the approximate sample label masks

\begin{tabular}{lll}
\hline Filter Type (kernel width) & Sampling $(\mathrm{mm})$ & Dilation $(\mathrm{mm})$ \\
\hline $1.7 \mathrm{~mm}$ gaussian blur & $1.0,0.7$ & 6.9 \\
$0.7 \mathrm{~mm}$ gaussian blur & $1.0,0.7$ & 5.2 \\
$0.7 \mathrm{~mm}$ gaussian blur on edge-detected images & 0.7 & 5.2 \\
none & 0.7 & 5.2 \\
\hline
\end{tabular}

As an illustration of registration protocol details, Table 1 specifies parameter values we used at $L_{1}$ level. $L_{1}$ consisted of 3 labels: head, upper body and lower body. In accordance with such coarse parcellation, we first downsampled both images by a factor of 3 in each dimension. The downsampling is important in two ways: (i) it simplifies the anatomy and in doing so it reduces the risk of trapping the optimizations in local minima, and (ii) it significantly speeds up the algorithm. The downsampled images were filtered in 3 different ways to enable a multi-scale/multi-resolution registration schedule.

To asses the accuracy of the registration we created a regular grid of points within the labeled region, totaling to 24042 points. The true displacements of the grid points, 
produced by the thin plate transformation, were compared to the displacements of the final deformation field $V_{5}$. Average error, measured by the distance between true and recovered grid point displacements was $0.08 \mathrm{~mm}$ ( $<1$ voxel), maximal error was $0.7 \mathrm{~mm}$ (approx. 6 voxels) and standard deviation of the error was $0.0005 \mathrm{~mm}$. With the exclusion of a tiny region near the edge of the mouse body, agreement was of subvoxel quality. The quality of agreement is illustrated in Fig. 4.
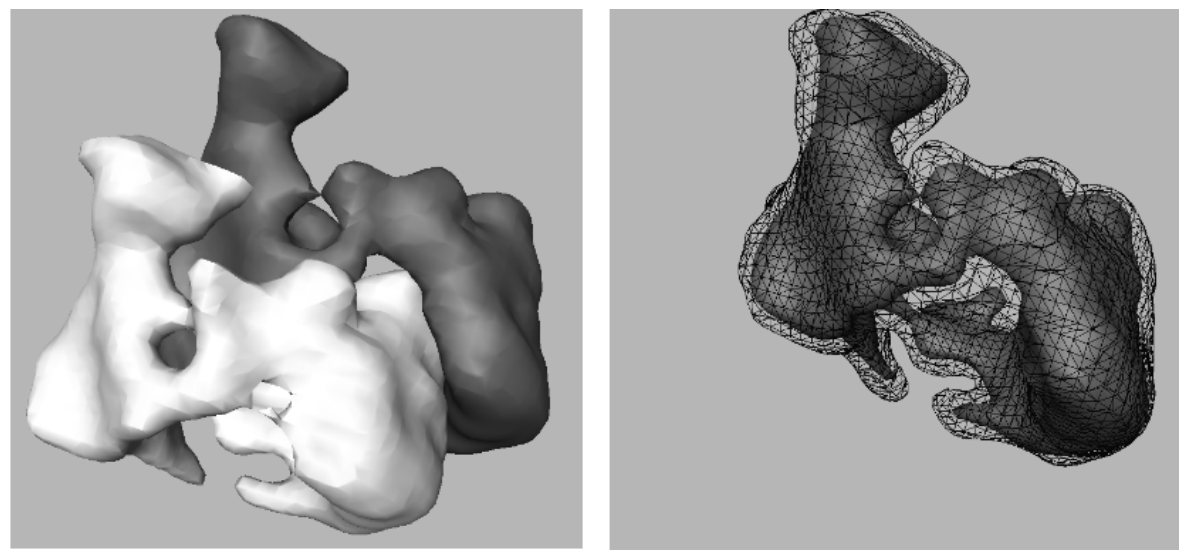

Fig. 4. Registration effect on a single vertebra. Left) The white surface shows the $18^{\text {th }}$ reference vertebra and the dark surface above it represents the same vertebra deformed by the synthetic transformation. Right) Once again, the dark surface represents the $18^{t h}$ synthetic vertebra while the wireframe shows the transformed white surface, according to the transformation produced by the algorithm (the wireframe surface is dilated by one voxel to enhance visual perception of the agreement). Note the position of the synthetic $18^{\text {th }}$ vertebra: it actually lies closer to the $17^{\text {th }}$ reference vertebra. Since neighboring vertebrae are almost identical in size and shape, the algorithm is challenged to establish the correct correspondence. However, the awareness of the hierarchical anatomy of the entire mouse body enables the algorithm to find the correct match, as demonstrated in the figure on the right

The algorithm executed in less than 1 hour using sixteen $400 \mathrm{MHz}$ processors. Considering that the images are quite large (1024x256x256 voxels), the achieved speed was excellent.

\section{Conclusions}

We have developed a methodology for fully automatic 3D registration of whole mouse body MR images. The deformation fields produced by the method are piecewise affine, where pieces correspond to anatomically defined ROI's based on a reference image. The accuracy of the registration has been assessed in a synthetic experiment where an a priori known deformation has been successfully recovered by the algorithm. The algorithm is inherently parallelizable, resulting in high computational efficiency. In our future work 
we intend to investigate further refinements of the method including regularization of the deformation fields and organ specific non-linear registrations.

Acknowledgments. The MR data used in the Results section, courtesy of Duke Center for In Vivo Microscopy (NCRR P41 05959, NCI R24 CA 92656).

\section{References}

1. A.W. Toga, ed.: Brain Warping. San Diego: Academic Press, 1999.

2. F.L. Bookstein: Principal warps:thin-plate splines and the decomposition of deformations. IEEE Trans. on Pattern Analysis and Machine Intelligence, 11(6):567-585, 1989.

3. D.L. Collins and A.C. Evans: Animal: Validation And Applications Of Non-Linear RegistrationBased Segmentation. International Journal and Pattern Recognition and Artificial Intelligence. 11, 1271-1294, Dec 1997.

4. J. Hajnal, D. Hawkes and D.Hill, ed.: Medical Image Registration. CRC Press, 2001.

5. P.M. Thompson, A.W. Toga: A Framework For Computational Anatomy. Comput. Visual. Sci. 5: 13-34 (2002)

6. R.P. Woods, S.T. Grafton, C.J. Holmes, S.R. Cherry, J.C. Mazziotta: Automated Image Registration: I. General Methods and Intrasubject, Intramodality Validation. Journal of Computer Assisted Tomography 1998;22:139-152.

7. J.A. Johnson, G.P. Cofer, S.L. Gewalt, L.W. Hedlund: Morphologic Phenotyping With MR Microscopy: The Visible Mouse. Radiology 2002, 222(3):789-793 